\title{
Vitamin D receptor deficiency increases systolic blood pressure by upregulating the renin-angiotensin system and autophagy
}

\author{
JIAN JIA $^{1}$, XU TAO $^{2}$, ZHOUNING TIAN $^{3}$, JING LIU $^{4}$, XIAOMAN YE $^{2}$ and YIYANG ZHAN ${ }^{2}$ \\ Departments of ${ }^{1}$ General Practice, ${ }^{2}$ Geriatric Medicine and \\ ${ }^{3}$ Cardiology, The First Affiliated Hospital of Nanjing Medical University; \\ ${ }^{4}$ The First School of Clinical Medicine, Nanjing Medical University, Nanjing, Jiangsu 210029, P.R. China
}

Received June 2, 2021; Accepted November 30, 2021

DOI: $10.3892 /$ etm.2022.11243

\begin{abstract}
The vitamin D receptor (VDR) may regulate blood pressure via multiple pathways. The present study investigated the underlying mechanism by which VDR deficiency increases blood pressure. A total of 16 8-week-old male littermate mice were randomly divided into the $V D R$ knockout and wild-type groups ( $V D R^{-/-}$and $V D R^{+/+}$, respectively). Blood pressure was measured using a four-channel PowerLab data acquisition and ADI software analysis system. After euthanasia, vascular smooth muscle cells (VSMCs) were isolated from the $V D R^{-1-}$ and $V D R^{+/+}$mice. Oxidative stress, renin-angiotensin system $(R A S)$ activation and autophagy markers were measured in the isolated VSMCs using reverse transcription-quantitative PCR (RT-qPCR), western blotting and transmission electron microscopy (TEM) assays. Mean systolic pressure was significantly higher in the $V D R^{-/-}$mice compared with the $V D R^{+/+}$mice. RT-qPCR and western blotting analyses indicated that RAS markers (angiotensin II and II type 1 receptor) were significantly upregulated, oxidative stress was increased (evidenced by reduced superoxide dismutase and peroxiredoxin-4) and autophagy was activated (upregulation of autophagy related protein 7 , Beclin 1 and microtubule-associated proteins 1A/1B light chain 3A) in the $V D R^{-/-}$VSMCs compared with the $V D R^{+/+}$VSMCs. TEM demonstrated that there were more autophagy bodies in the $V D R^{-/-}$VSMCs compared with the $V D R^{+/+}$VSMCs. In conclusion, VDR deficiency was associated with high blood pressure. The mechanism underlying the increase in blood pressure caused by VDR deficiency may involve activation of the RAS, as well as increased oxidative stress and autophagy of VSMCs.
\end{abstract}

Correspondence to: Dr Yiyang Zhan, Department of Geriatric Medicine, The First Affiliated Hospital of Nanjing Medical University, 300 Guangzhou Road, Nanjing, Jiangsu 210029, P.R. China

E-mail: yiyangzhan@sina.com

Key words: vitamin D receptor, hypertension, vascular smooth muscle cell, oxidative stress, autophagy

\section{Introduction}

Vitamin D signaling plays a role in regulating blood pressure through influencing vascular endothelial function, oxidative stress and activation of the renin-angiotensin-system (RAS), as well as increasing insulin resistance. The widespread effect of vitamin $\mathrm{D}$ relies on the extensive presence of the vitamin D receptor (VDR), which is expressed in every human tissue and nearly all nucleated cells, although at varying levels. It is currently hypothesized that almost all biological actions of vitamin $\mathrm{D}$ are mediated by its active form, $1,25(\mathrm{OH})_{2} \mathrm{D}$, signaling mainly through the intracellular VDR (1). Vitamin D signaling has been associated with elevated plasma renin and angiotensin (Ang) II levels (2). Animal experiments have indicated that $1,25(\mathrm{OH})_{2} \mathrm{D}_{3}$ can inhibit renin gene transcription (3), and Zhou et al (4) revealed that blood pressure is higher in $1 \alpha(\mathrm{OH})$ ase $^{-/-}$mice compared with wild-type mice, which is accompanied by elevated $m R N A$ expression levels of renin, plasma aldosterone and Ang II. These studies indicate that $1,25(\mathrm{OH})_{2} \mathrm{D}_{3}$ may influence blood pressure by regulating the central and peripheral RAS through an anti-oxidative stress mechanism. Another in vivo experiment (5) suggested that vitamin D deficiency increases Ang II and oxygen anion levels in local vascular smooth muscle cells (VSMCs). Several studies have also demonstrated that $1,25(\mathrm{OH})_{2} \mathrm{D}_{3}$ deficiency can increase blood pressure by inducing oxidative stress pathways and over-activating the central RAS (6-8).

When vitamin D levels are adequate, a number of the intracellular oxidative stress-related activities are downregulated. Suboptimal concentrations of serum 25(OH)D fail to subdue oxidative stress conditions, augment intracellular oxidative damage and decrease the rate of apoptosis. Superoxide dismutase (SOD) belongs to a group of antioxidant enzymes that play a significant role in regulating oxidative stress in cells (9). Peroxiredoxin 4(Prdx4), a typical endoplasmic reticulum-resident 2-Cys antioxidant of peroxiredoxins, can fine-tune hydrogen peroxide catabolism, which affects cell survival by affecting redox balance, oxidative protein folding and hydrogen peroxide signaling (10). Vitamin D can regulate autophagy at different levels, including induction, nucleation and elongation to maturation and degradation, which affects the occurrence and development of diseases (11). 
We previously investigated the association between $V D R$ gene polymorphisms and hypertension. We revealed polymorphisms in VDRrs11574129, rs2228570 and rs739837 in 2,409 patients with hypertension and 3,063 controls, and that the $r s 228570$ polymorphism is significantly correlated with risk of hypertension (12). However, the mechanism by which vitamin $\mathrm{D}$ signaling regulates blood pressure remains unclear. Therefore, the present study established a $V D R$ deficiency animal model using $V D R$ knockout mice to investigate how VDR regulates blood pressure.

\section{Materials and methods}

Animals. $V D R^{-/-}$mice were derived by homologous recombination in embryonal stem cells as described previously (gifted from Dr Marie Demay; Massachusetts General Hospital, MA, USA) (13). $V D R$ wild-type $\left(V D R^{+/+}\right)$and knockout $\left(V D R^{-/}\right)$ mice were identified using western blotting analysis of tail blood samples (Fig. S1). Animal experiments were approved by the Institutional Animal Care and Use Committee of Nanjing Medical University (approval no. IACUC-1910005). All procedures performed in studies involving animals were in accordance with the ethical standards of the institution or practice at which the studies were conducted. For sample preparation, $100 \%$ carbon dioxide was used to euthanize the animals (14).

Experimental mice were raised in the SPF Animal Center of Nanjing Medical University, where the room temperature was maintained at $20-24^{\circ} \mathrm{C}$ and $\sim 60 \%$ humidity with a $12 \mathrm{~h}$ light/dark schedule. A total of 168 -week-old male littermates were randomly divided into the $V D R^{+/+}$and $V D R^{-/}$groups $(\mathrm{n}=8$ mice per group). After weaning, the mice were fed a regular diet or a 'rescue diet' (Harlan Teklad; Envigo) containing 20\% lactose, $1.25 \%$ phosphorus and $2 \%$ calcium for 8 weeks.

Blood pressure measurements. The ML125 non-invasive blood pressure (NIBP) system (AD Instruments) was used to measure systolic blood pressure in conscious animals. A pneumatic pulse sensor cuff was placed on the tails. After habituation to this setting for 7 days, systolic blood pressure was recorded. To obtain accurate blood pressure recordings, the mice were kept in a motionless and undisturbed state during the measurement. Conditioning was achieved once the mice were processed gently without forcing restraint. Systolic blood pressure was recorded consecutively for 3 days in chambers that were maintained at $31-33^{\circ} \mathrm{C}$. Systolic blood pressure was recorded separately in $10 \mathrm{~min}$ intervals over a total of 10 recordings. The average measurement was calculated for the 10 recordings.

VSMC culture. Primary VSMCs were isolated from aortas of 6- to 8-week-old mice. The mice were euthanized using $\mathrm{CO}_{2}$ (14). After removal of the adventitia, the aorta was opened to expose the endothelial layer under a dissection microscope. Tissues from 6 to 8 animals were pooled and incubated with trypsin $(0.25 \% \mathrm{w} / \mathrm{v})$ at room temperature for $10 \mathrm{~min}$ to remove any remaining adventitia and endothelium. Tissues were incubated overnight in $\alpha$ Minimum Essential Medium ( $\alpha$ MEM) supplemented with $10 \%$ fetal calf serum, $100 \mathrm{U} / \mathrm{ml}$ penicillin, $100 \mu \mathrm{g} / \mathrm{ml}$ streptomycin and $0.25 \mu \mathrm{g} / \mathrm{ml}$ amphotericin (complete $\alpha \mathrm{MEM}$ ) at room temperature before being digested with $425 \mathrm{U} / \mathrm{ml}$ collagenase type II (Worthington Biochemical Corporation) for $5 \mathrm{~h}$ at room temperature. Isolated VSMCs were expanded in T25 tissue culture flasks in a humidified atmosphere with $5 \% \mathrm{CO}_{2}$ at $37^{\circ} \mathrm{C}$ until reaching confluence. VSMCs were identified by negative platelet endothelial cell adhesion molecule1 (marker of endothelial cells) and vimentin (marker of fibroblasts), and positive $\alpha$-smooth muscle actin (marker of VSMCs) expression. VSMCs from the 3rd to 5th passages were used in the present study (15).

Reverse transcription-quantitative PCR (RT-qPCR). TRIzol ${ }^{\circledR}$ reagent (Invitrogen; Thermo Fisher Scientific, Inc.) was used to isolate total RNA from VSMCs according to the manufacturer's instructions. $m R N A$ levels of SOD, Ang $I I$ and Ang II type 1 receptor (ATIR) in the VSMCs were detected using RT-qPCR (15). Briefly, first-strand $c D N A s$ were reverse-transcribed from $2 \mu \mathrm{g}$ of total RNAs using M-MLV (Moloney Murine Leukemia Virus Reverse Transcriptase) with oligo $(d T)$; as the primer) and Rain in $1 \mathrm{X}$ M-MLV buffer. Each $1 \mu \mathrm{l}$ of the $c D N A$ was then applied as a template for the PCR amplification using the SYBR ${ }^{\circledR}$ Green PCR reagent kit (Toyobo Life Science) in a PCR cycler (Applied Biosystems; Thermo Fisher Scientific, Inc.). GAPDH expression was applied as a loading reference. The target mRNA was amplified in the following thermocycling conditions: initial denaturation for $10 \mathrm{~min}$ at $95^{\circ} \mathrm{C}, 40$ cycles of denaturation for $15 \mathrm{sec}$ at $95^{\circ} \mathrm{C}$, annealing for $40 \mathrm{sec}$ at $55^{\circ} \mathrm{C}$, extension for $30 \mathrm{sec}$ at $72^{\circ} \mathrm{C}$ and final extension for $7 \mathrm{~min}$ at $72^{\circ} \mathrm{C}$. The following PCR primers were used for corresponding gene detection: Ang-II (NC_000023.11) forward primer: 5'-CCT CCCGACTAGATGGACAC-3'and reverse primer: 5'-GAG GGCAGGGGTAAAGAGAG-3'; AT1R (NC_000003.12) forward primer: 5'-ATGTTTCTTGGTGGCTTGGT-3' and reverse primer: 5'-CCTGAGAGGGTCCGAAGAAA-3'; SOD1 (NC_000021.9) forward primer: 5'-AACCATCCACTT CGAGCAGA-3' and reverse primer: 5'-GGTCTCCAACAT GCCTCTCT-3'; GAPDH forward primer: 5'-GAACGGGAA GCTCACTGG-3' and reverse primer: 5'-GCCTGCTTCACC ACCTTCT-3'. Relative expression level of the respective target gene was calculated according to the $2^{-\Delta \Delta \mathrm{Cq}}$ method (16).

Western blotting. VSMCs were lysed in RIPA buffer (Sigma-Aldrich; Merck KGaA) containing proteinase inhibitors (Sigma-Aldrich). The protein concentration of the lysate was determined using a BCA kit (Merck KGaA). Aliquots $(20 \mu \mathrm{g})$ of the extracted protein sample were boiled for $5 \mathrm{~min}$, loaded on an $10 \%$ SDS-PAGE gel, separated by electrophoresis and then transferred onto a nitrocellulose membrane. The membrane was blocked with $5 \%$ milk at room temperature in phosphate-buffered saline/0.05\% Tween 20 (PBST) for $3 \mathrm{~h}$ and then incubated with a monoclonal antibody against renin (1:1,000; cat. no. 70R-1584; Fitzgerald) overnight at $4^{\circ} \mathrm{C}$. After three washes with PBST, the membrane was incubated with horseradish peroxidase-conjugated secondary antibodies (1:1,000; cat. no. A0208; Beyotime Institute of Biotechnology) at room temperature for $1 \mathrm{~h}$. Finally, the probed bands were visualized using an Enhanced Chemiluminescence reagent (PerkinElmer) and analyzed using ImageJ (version 1; National Institutes of Health) (15). Other used antibodies were as 
follows: anti-LC3-A (cat. no. AF5225; 1:1,000), anti-Beclin1 (cat.no. AF5123; 1:1,000), anti-AGT7 (cat.no. AA820; 1:1,000), and anti-GAPDH (cat. no. AF0006; 1:1,000) were purchased from Beyotime Institute of Biotechnology.Anti-ATR1 (cat. co. PB0492; 1:1,000), anti-P62 (cat. no. BA2849; 1:1,000), and anti-PRDX4 (cat. co. PB9383; 1:1,000) were purchased from BOSTER Institute of Biotechnology. GAPDH was used as a loading control.

Transmission electron microscopy (TEM). For cellular TEM observation, VSMCs were cultured for $120 \mathrm{~min}$ and then fixed with $2.5 \%$ glutaraldehyde and post-fixed with $3 \%$ osmium tetroxide for $2 \mathrm{~h}$ at room temperature. The specimen was dehydrated in a graded series of ethanol, embedded with EPon812, and stained by uranium acetate and aluminum citratein. Epon resin and then observed with a Hitachi-600 TEM (Hitachi, Ltd.) to evaluate the formation of autophagosomes in the cells (17).

Statistical analysis. All data were statistically analyzed using SPSS software (version 13.0; SPSS, Inc.). In the present study, all data are presented as the mean \pm standard deviation. Average data between the groups was compared using the unpaired Student's t-test. The Levene test was applied for distribution analysis. $\mathrm{P}<0.05$ was considered to indicate a statistically significant difference.

\section{Results}

Systolic blood pressure is elevated in $V D R^{-/-}$mice. Systolic blood pressure was measured using the NIBP system in all mice. The systolic blood pressure of the $V D R^{-/-}$mice was significantly higher compared with the $V D R^{+/+}$littermate control mice (Fig. 1).

The RAS is upregulated in VDR ${ }^{-/-}$mice. To understand how $V D R$ deficiency affects hypertension and the RAS, the expression levels of RAS factors Ang II and AT1R were measured using western blotting and RT-qPCR assays in VSMCs isolated from both $V D R^{-/}$and $V D R^{+/+}$mice. Protein expression of AT1R was significantly increased in the $V D R^{-/-} \mathrm{VSMCs}$ compared with the $V D R^{+/+}$VSMCs (Fig. 2A). The mRNA levels of Ang II and ATIR were significantly upregulated in the $V D R^{-/-}$VSMCs compared with the $V D R^{+/+}$VSMCs (Fig. 2B). These results suggested that deletion of VDR upregulated the RAS in mice.

Oxidative stress is elevated in $V D R^{-/-}$mice. The association between hypertension in the $V D R^{-/-}$mice and oxidative stress levels were determined in the VSMCs. mRNA levels of $S O D$ were measured using RT-qPCR and protein expression of Prdx4 was measured using western blotting analysis in the VSMCs isolated from both $V D R^{-/-}$and $V D R^{+/+}$mice. The results demonstrated that the mRNA levels of $S O D$ were significantly downregulated in the $V D R^{-/-}$VSMCs compared with the $V D R^{+/+} \mathrm{VSMCs}$ (Fig. 3A). The protein expression of Prdx 4 was significantly decreased in the $V D R^{-/-} \mathrm{VSMCs}$ compared with the $V D R^{+/+}$VSMCs (Fig. 3B). These data indicated that $V D R$ deficiency upregulated oxidative stress in mice.

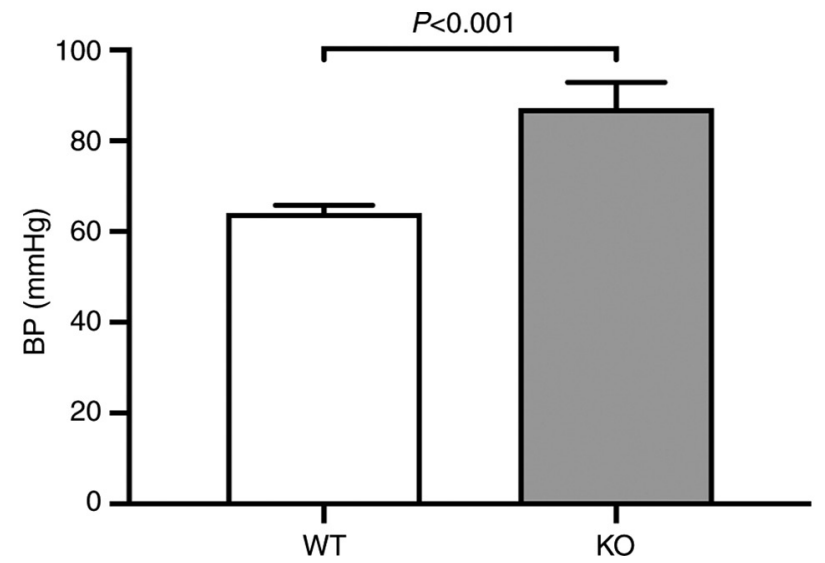

Figure 1. Systolic blood pressure of the $V D R^{-/-}$and $V D R^{+/+}$mice. Average systolic blood pressure was calculated from 10 measurements using the NIBP non-invasive blood pressure system in the KO and WT mice. BP, blood pressure; $\mathrm{KO}$, knockout $/ V D R^{-/} ; \mathrm{WT}$, wild-type $/ V D R^{+/+} ; V D R$, vitamin D receptor.

Protein expression levels of autophagy-related factors are upregulated in VSMCs of $V D R^{-/}$mice. The expression levels of autophagy-related factors, including autophagy-related protein 7 (ATG7), Beclin1, microtubule-associated proteins 1A/1B light chain 3A(LC3A) and nucleoporin p62 (p62), were measured in the VSMCs of $V D R^{-/-}$and $V D R^{+/+}$mice using western blotting analysis. ATG7, Beclin1 and LC3Awere significantly upregulated, while p62 was significantly downregulated in the $V D R^{-/-}$VSMCs compared with the $V R D^{+/+}$ VSMCs (Fig. 4).

TEM reveals increased autophagosomes in VSMCs of $V D R^{-/-}$mice. Next, the VSMC ultrastructure in the $V D R^{-/-}$and $V D R^{+/+}$mice was analyzed using TEM. An increased number of autophagy bodies were observed in the $V D R^{-/-} \mathrm{VSMCs}$ compared with the $V D R^{+/+}$VSMCs (Fig. 5). These results suggested that $V D R$ deficiency could activate autophagy in VSMCs.

\section{Discussion}

Oxidative stress can injure blood vessels and serve as a pathogenic factor in hypertension. Numerous studies have demonstrated that there is an imbalance between the anti-oxidative defense system and the production of oxygen free radicals, causing a high level of oxidative stress in patients with hypertension (18-20). Dysfunction of vascular endothelial cells caused by oxidative stress is considered to be the main cause of hypertension (21). Oxidative stress is closely associated with endothelial cell inflammation, hypertrophy, apoptosis, migration, fibrosis and vascular remodeling in hypertension $(19,22)$.

Since the VDR is widely distributed in vascular endothelial cells, VSMCs and cardiomyocytes, the role of VDR in hypertension has received extensive attention. In a previous observational study, activation of the VDR is associated with lower cardiovascular risk and improved survival (23). VDR deficiency can elevate intracellular oxidative stress (23), and VDR agonists have been demonstrated to synergistically alleviate diabetic atherosclerosis 
A

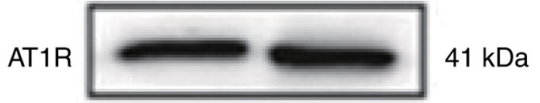

GAPDH
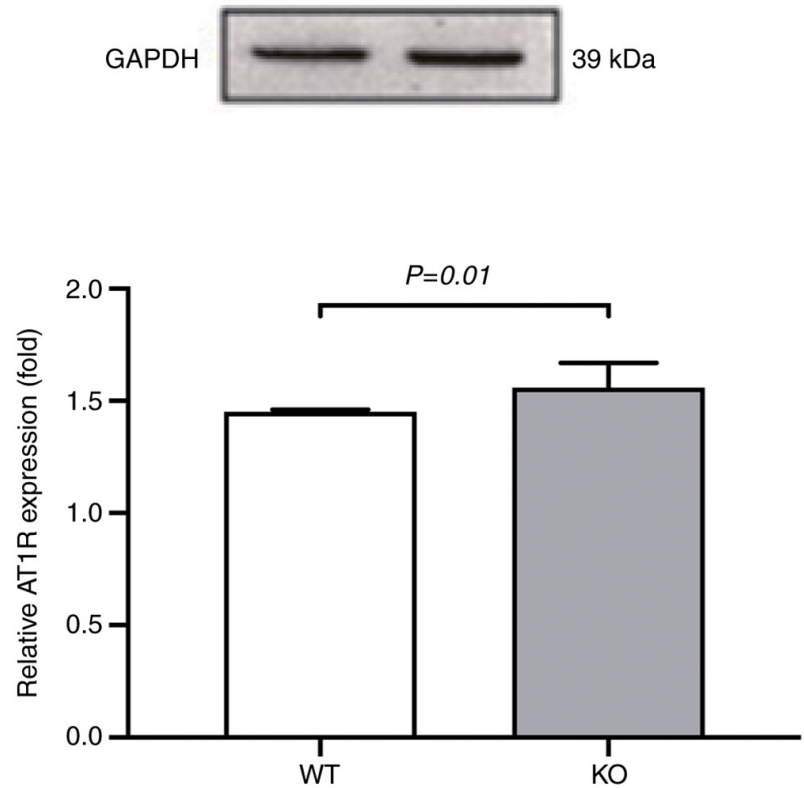

B
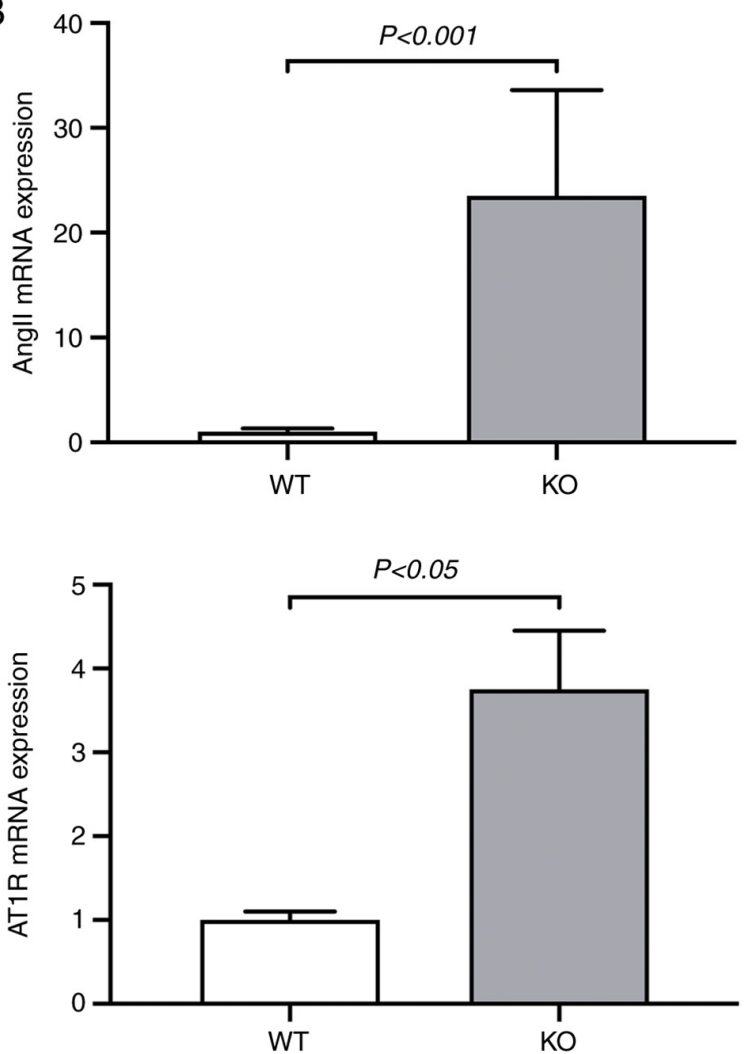

Figure 2. Ang II and AT1R expression levels in VSMCs of VDRKO mice. (A) Protein expression levels of AT1R in the VSMCs isolated from VDRKO and $V D R W T$ mice were detected using western blotting analysis and quantified. (B) mRNA levels of $A n g I I$ and $A T 1 R$ in the VSMCs isolated from $K O$ and WT mice were analyzed using reverse transcription-quantitative PCR. The data are presented as an average from three independent assays. WT levels were set at 1.0 for data normalization. $K O$, knockout $/ V D R^{-/}$; WT, wild-type/VDR ${ }^{+/+}$; Ang, angiotensin; AT1R, Ang II type 1 receptor; VSMCs, vascular smooth muscle cells; VDR, vitamin D receptor.

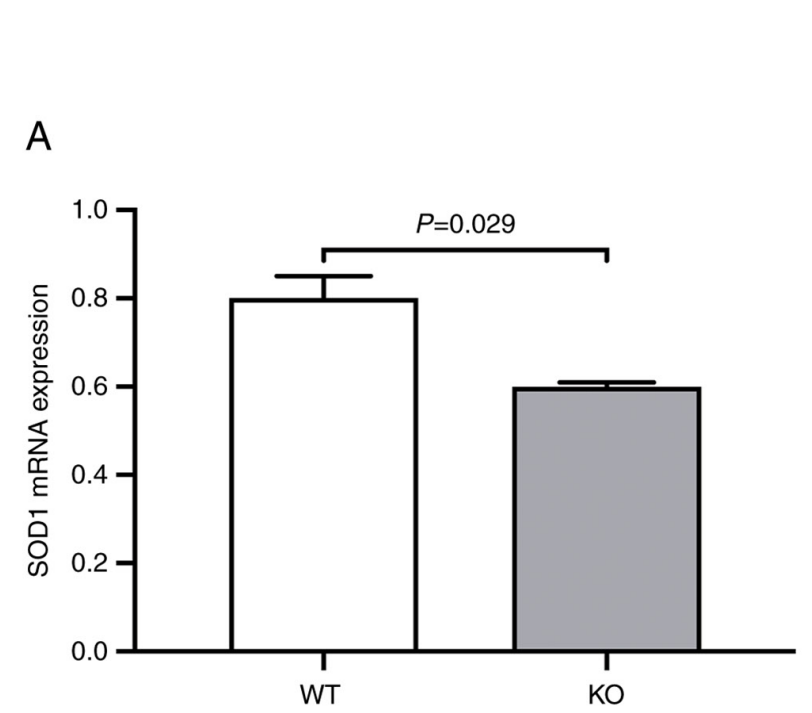

B
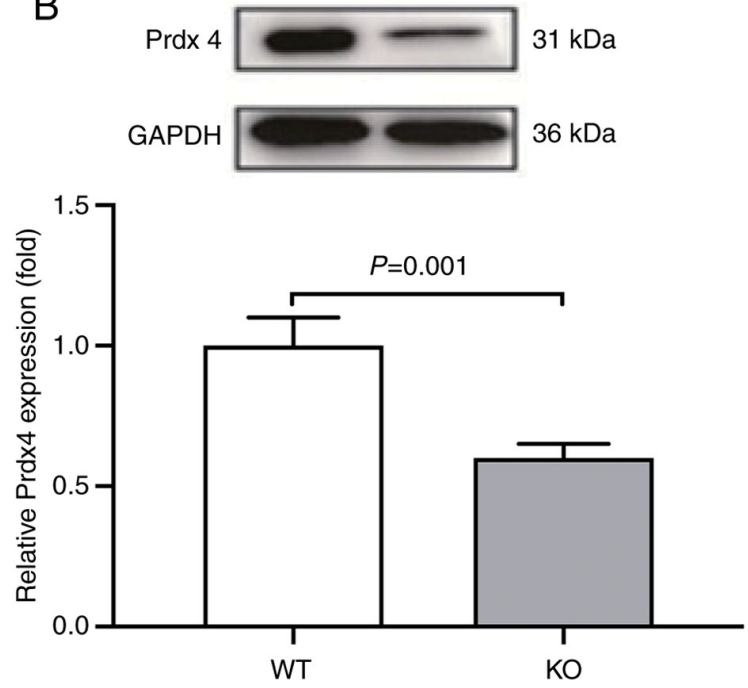

Figure 3. Expression levels of SOD and Prdx4 in VSMCs of $V D R^{-/}$mice. (A) mRNA levels of SOD in the VSMCs isolated from the $V D R K O$ and $V D R W T$ mice were analyzed using reverse transcription-quantitative PCR. The average intensity of the corresponding mRNA levels of the genes was calculated from three independent assays. (B) Protein expression of Prdx4 in the samples at the same conditions as A was detected using western blotting analysis. The average data are from three independent assays. WT levels were set at 1.0 for data normalization. SOD, superoxide dismutase; Prdx4, peroxiredoxin4; KO, knockout/VDR $R^{-/}$; WT, wild-type/VDR ${ }^{+/+}$; VSMCs, vascular smooth muscle cells; VDR, vitamin D receptor.

by inhibiting oxidative stress (24). Consistent with previous findings, the present study revealed that SOD mRNA levels and $\operatorname{Prdx} 4$ protein expression were significantly downregulated in $\mathrm{VDR}^{-/-}$mice compared with the $V D R^{+/+}$ mice. These data suggested that VDR deficiency could increase oxidative stress. 
A

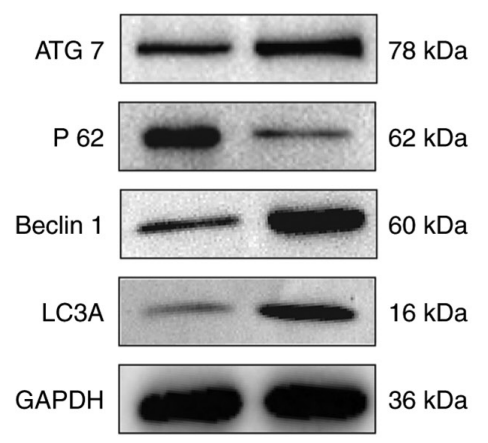

C

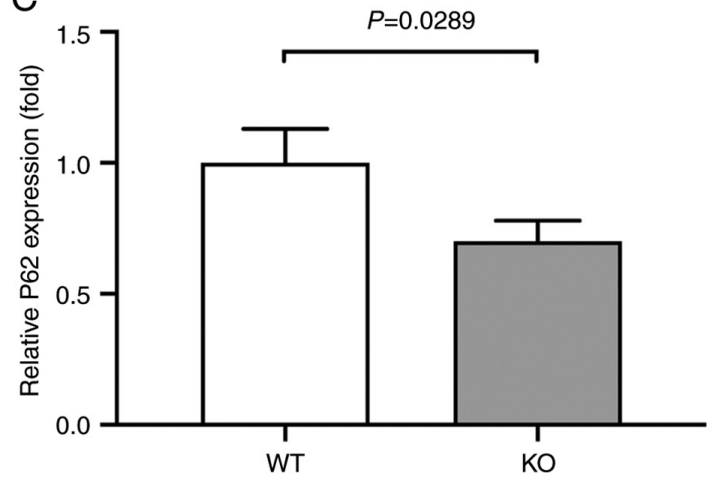

B

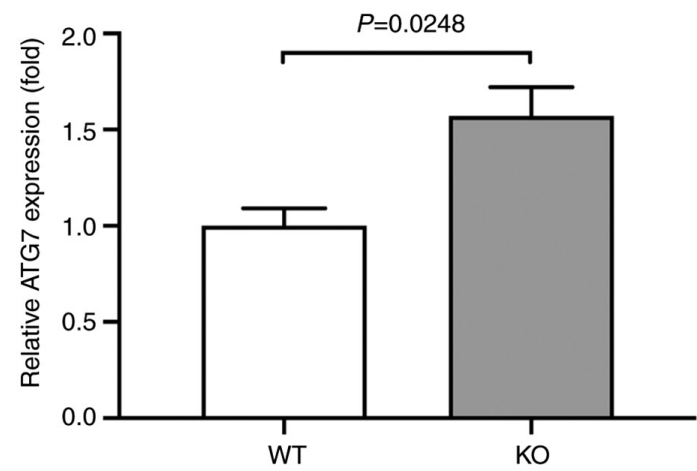

D

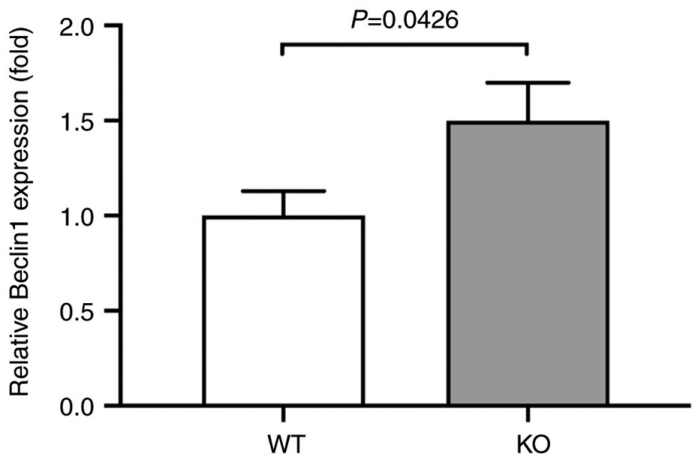

E

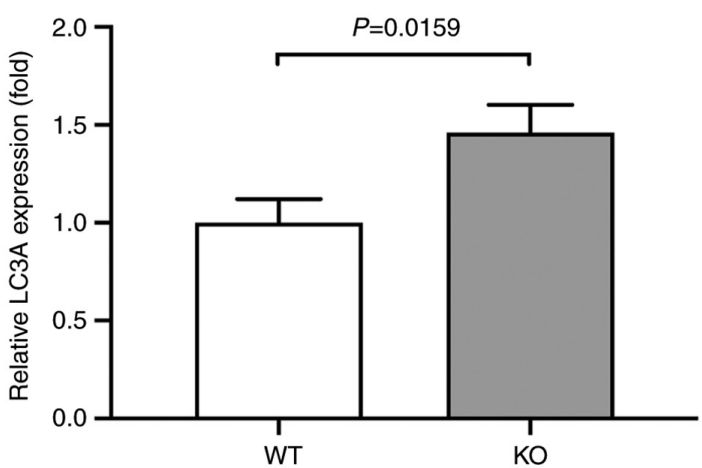

Figure 4. Protein expression of ATG7, Beclin1, LC3A and p62 in VSMCs. Protein expression of ATG7, Beclin1, LC3A and p62 in VSMCs isolated from $V D R K O$ and $V D R W T$ mice was detected using western blotting analysis. (A) Representative images of the corresponding western blot images. The average intensities of (B) ATG7, (C) p62, (D) Beclin1 and (E) LC3A are summarized in the corresponding bar graphs from three independent assays. WT levels were set at 1.0 for data normalization. KO, knockout/VDR ${ }^{-/}$; WT, wild-type/VDR ${ }^{+/+}$; VSMCs, vascular smooth muscle cells; ATG7, autophagy-related protein 7; p62, nucleoporin $p 62$; LC3A, microtubule-associated proteins 1A/1B light chain 3A; VDR, vitamin D receptor.

Oxidative stress reflects an imbalance between the reactive oxygen species and a biological ability to detoxify or repair the resulting damage. SOD is an enzyme that downregulates $\mathrm{O}^{2}$-byscavenging potentially damage-free radical moieties. It acts as a major anti-oxidative enzyme in almost all organisms. Therefore, SOD level reflects the anti-oxidative capacity. The higher the level of SOD, the higher capacity of anti-oxidation, which results the positive balance of anti-oxidation and pro-oxidation. Oppositely, the lower level of the SOD, the lower capacity of anti-oxidation, which causes the negative balance of anti-oxidation and pro-oxidation or oxidative damage (23). The results of the present study revealed that SOD level was decreased in the $V D R^{-/}$mice, which indirectly reflects the upregulated oxidative stress.

Indeed, this pattern is consistent with a number of previous findings. For example, in primary angle closure glaucoma, oxidative stress is increased accompanied with a decrease of
SOD level (25). Under normal circumstances, production and clearance of reactive oxygen species (ROS) are in equilibrium. However, once ROS production exceeds clearance, a large number of oxygen free radicals will be generated in the body. In patients with hypertension, increased production of ROS results in decreased levels of SOD, destruction of unsaturated fatty acids and increased lipid peroxidation, causing increased production of malondialdehyde.

Vitamin D signaling plays an important role in the inhibition of renin secretion and synthesis. Disruption of VDR signaling transduction leads to RAS activation, cardiac hypertrophy and hypertension (26). It has been demonstrated that $V D R^{-/-}$mice, a model of vitamin D signal disruption, develop hypertension (26). Vitamin D inhibits the renin-angiotensin-aldosterone system by blocking renin gene expression (3). Plasma renin and Ang II levels are negatively correlated with $1,25(\mathrm{OH})_{2} \mathrm{D}_{3}(27)$. Knockout of VDR and cytochrome P450 27B1 in mice results 

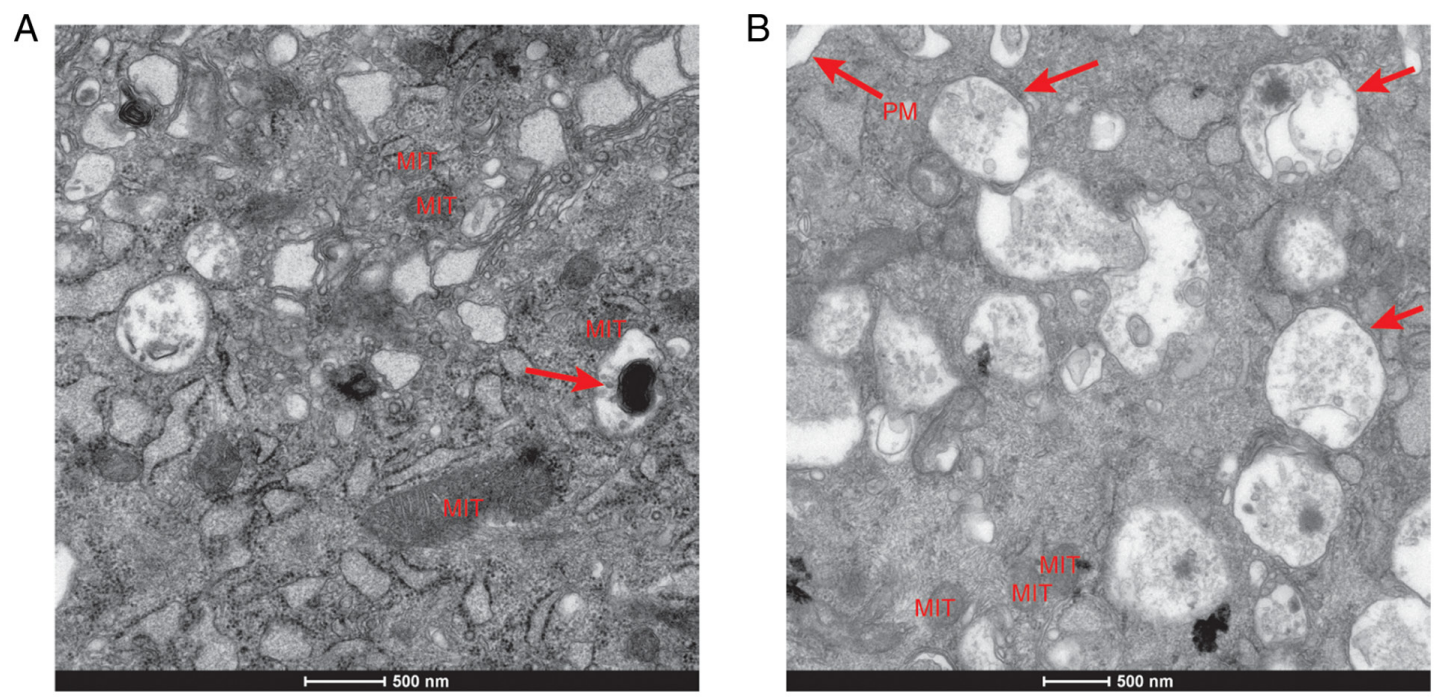

Figure 5. Ultrastructural alterations in VSMCs of $V D R^{-\digamma}$ mice. Changes in VSMC ultrastructure in the (A) VDRWT and (B) VDRKO mice were examined using TEM (scale bar, $500 \mathrm{~nm}$ ). Autophagy bodies are indicated by the red arrows. PM, plasma membrane; VDR, vitamin D receptor; VSMCs, vascular smooth muscle cells; KO, knockout/VDR

in elevated serum renin and RAS activity and increased blood pressure (5). Xiang et al (28) reported an increase in renin and Ang II mRNA levels in the hearts of $1 \alpha(\mathrm{OH})$ ase and $V D R$ knockout mice. The present study demonstrated that AT1R and Ang II levels increased significantly in $V D R^{-/}$VSMCs, consistent with the previous findings. Vitamin $\mathrm{D}$ can inhibit renin gene expression by activating the cAMP response elements at the promoter region of the renin gene (4). Overexpression of VDR can inhibit renin production in renal par acyclic cells (29). Based on the result of the present study and the literature, we hypothesize that $V D R$ deficiency induces overexpression of renin, thus activating the RAS in mouse VSMCs. However, further research is needed to confirm this hypothesis.

Autophagy plays an important role in human health. Numerous disorders are associated with autophagy imbalances, such as hypertension and cardiac disease (30). Vitamin $\mathrm{D}$ has been reported to regulate autophagy through multiple pathways, including gene induction, nucleation and elongation of protein maturation and degradation (31). However, the mechanism by which the VDR regulates autophagy has not been fully determined. An improved understanding of this mechanism could be useful for clinical diagnosis and treatment of relative diseases.

To the best of our knowledge, thus far, studies on vitamin D-mediated regulation of autophagy have mainly focus on the phosphatidylinositol 3 kinase/Beclin-1 pathway, $\mathrm{Ca}^{2+}$ levels, toll-like receptor signaling pathway, antimicrobial peptides and lysosomes, autophagy related gene expression and inflammatory factors (31). Ang II, a vasoactive peptide, plays a notable role in numerous vascular disorders. An imbalance in vascular autophagy, excessive VSMC proliferation and vascular remodeling can lead to increased vascular resistance and lumen stenosis, resulting in increased blood pressure $(32,33)$. Hypertensive rats demonstrated endothelial dysfunction in aortic and mesenteric arteries, with decreased phosphorylated (p)-Akt, p-mTOR and autophagy marker protein p62, and increased LC3 II/I levels (34). Ang II and glomerular podocyte autophagic activity increased significantly in hypertensive rat kidneys; therefore, high blood pressure caused by kidney injury may be associated with Ang II-induced glomerular podocyte autophagy. Excessive autophagy causes endothelial dysfunction in rats, but it has been revealed that endothelial function can be improved and blood pressure can be reduced through regulating autophagy (35). Ang II induces autophagy through the IAT1R/Rhoda/Rho kinase pathway, causing hypertrophy of VSMCs (36). The interaction between autophagy, oxidative stress and the RAS plays a notable role in vascular remodeling and vascular damage caused by hypertension $(37,38)$.

The objective of the present study was to explore the possible mechanism of VDR deficiency on the RAS and cellular autophagy in a mouse model of vitamin $\mathrm{D}$ deficiency. The present study data demonstrated that VDR deficiency increased oxidative stress via downregulating SOD levels and $\operatorname{Prdx} 4$ expression, and activating autophagy via upregulation of ATG7, Beclin1 and LC3Aexpression levels in VSMCs. We hypothesize that the increased autophagy level induced by $V D R$ deficiency may be associated with activation of the RAS and signaling pathways downstream of oxidative stress.

In conclusion, the present study suggested that $V D R$ deficiency increased blood pressure by elevating oxidative stress factors and RAS activity, in addition to causing excessive autophagy of VSMCs. The present study offered novel insight into the mechanism by which VDR regulates blood pressure and provided theoretical evidence to guide clinicians on administering $1,25(\mathrm{OH})_{2} \mathrm{D}_{3}$ for the prevention and treatment of hypertension.

\section{Acknowledgements}

The authors thank Dr Marie Demay (Massachusetts General Hospital, MA, USA) for supplying the $V D R^{-/-}$mice for this study.

\section{Funding}

This study was supported by Young Scholars Fostering fund of the First Affiliated Hospital of Nanjing Medical University 
(PY2021015); Jiangsu Province 'Six Talent Peaks' High-Level Talent Project (grant no. WSN-024); and Jiangsu Research Center for Primary Health Development and General Practice Education (grant no. 2019B03).

\section{Availability of data and materials}

The datasets used and/or analyzed during the current study are available from the corresponding author on reasonable request.

\section{Authors' contributions}

YZ performed the research conception and design. XT, JL, YZ and XY performed the experiments. JJ and ZT analyzed and checked the data, and drafted the manuscript. JJ, ZT and XY prepared figures. JJ and YZ edited and revised manuscript. YZ was primarily responsible for final content. JJ and YY confirm the authenticity of all the raw data. All authors read and approved the final manuscript.

\section{Ethics approval and consent to participate}

Animal experiments were approved by the Institutional Animal Care and Use Committee of Nanjing Medical University (approval no. IACUC-1910005). All procedures performed in studies involving animals were in accordance with the ethical standards of the institution or practice at which the studies were conducted.

\section{Patient consent for publication}

Not applicable.

\section{Competing interests}

The authors declare that they have no competing interests.

\section{References}

1. Latic N and Erben RG: Vitamin D and cardiovascular disease, with emphasis on hypertension, atherosclerosis, and heart failure. Int J Mol Sci 21: 6483, 2020.

2. Tamez H, Kalim S and Thadhani RI: Does vitamin D modulate blood pressure? Curr Opin Nephrol Hypertens 22: 204-209, 2013.

3. Yuan W, Pan W, Kong J, Zheng W, Szeto FL, Wong KE, Cohen R, Klopot A, Zhang Z and Li YC: 1,25-dihydroxyvitamin D3 suppresses renin gene transcription by blocking the activity of the cyclic AMP response element in the renin gene promoter. J Biol Chem 282: 29821-29830, 2007.

4. Zhou C, Lu F, Cao K, Xu D, Goltzman D and Miao D: Calcium-independent and 1,25(OH)2D3-dependent regulation of the renin-angiotensin system in lalpha-hydroxylase knockout mice. Kidney Int 74: 170-179, 2008.

5. Andersen LB, Przybyl L, Haase N, von Versen-Höynck F, Qadri F, Jørgensen JS, Sorensen GL, Fruekilde P, Poglitsch M, Szijarto I, et al: Vitamin D depletion aggravates hypertension and target-organ damage. J Am Heart Assoc 4: e001417, 2015.

6. Ji S, Doumit ME and Hill RA: Regulation of adipogenesis and key adipogenic gene expression by 1,25 -dihydroxyvitamin $\mathrm{D}$ in 3T3-L1 cells. PLoS One 10: e0126142, 2015.

7. Yin Y, Yu Z, Xia M, Luo X, Lu X and Ling W: Vitamin D attenuates high fat diet-induced hepatic steatosis in rats by modulating lipid metabolism. Eur J Clin Invest 42: 1189-1196, 2012.

8. Asano L, Watanabe M, Ryoden Y, Usuda K, Yamaguchi T, Khambu B, Takashima M, Sato SI, Sakai J, Nagasawa K and Uesugi M: Vitamin D metabolite, 25-hydroxyvitamin D, regulates lipid metabolism by inducing degradation of SREBP/SCAP. Cell Chem Biol 24: 207-217, 2017.
9. Wimalawansa SJ: Vitamin D deficiency: Effects on oxidative stress, epigenetics, gene regulation, and aging. Biology (Basel) 8: 30, 2019.

10. Fujii J, Ikeda Y, Kurahashi T and Homma T: Physiological and pathological views of peroxiredoxin 4. Free Radic Biol Med 83: 373-379, 2015.

11. Liu L, Li J, Deng C and Chen D: Advances in the mechanism of vitamin D affecting autophagy. Zhonghua Wei Zhong Bing Ji Jiu Yi Xue 30: 1103-1106, 2018 (In Chinese).

12. Jia J, Shen C, Mao L, Yang K, Men C and Zhan Y: Vitamin D receptor genetic polymorphism is significantly associated with decreased risk of hypertension in a Chinese Han population. J Clin Hypertens (Greenwich) 16: 634-639, 2014.

13. Li YC, Pirro AE, Amling M, Delling G, Baron R, Bronson R and Demay MB: Targeted ablation of the vitamin $D$ receptor: An animal model of vitamin D-dependent rickets type II with alopecia. Proc Natl Acad Sci USA 94: 9831-9835, 1997.

14. Boivin GP, Hickman DL, Creamer-Hente MA, Pritchett-Corning KR and Bratcher NA: Review of $\mathrm{CO}_{2}$ as a euthanasia agent for laboratory rats and mice. J Am Assoc Lab Anim Sci 56: 491-499, 2017.

15. Patel JJ, Bourne LE, Millán JL, Arnett TR, MacRae VE, Wheeler-Jones CPD and Orriss IR: Inhibition of vascular smooth muscle cell calcification by ATP analogues. Purinergic Signal 15: 315-326, 2019.

16. Livak KJ and Schmittgen TD: Analysis of relative gene expression data using real-time quantitative PCR and the 2(-Delta Delta C(T)) method. Methods 25: 402-408, 2001.

17. Zhang J, Yuan L, Wang S, Liu J, Bi H, Chen G, Li J and Chen L: Germacrone protects against oxygen-glucose deprivation/reperfusion injury by inhibiting autophagy processes in PC12 cells. BMC Complement Med Ther 20: 77, 2020.

18. Allison SJ: Hypertension: Oxidative stress and immune activation in hypertension. Nat Rev Nephrol 12: 4, 2016.

19. Sinha N and Dabla PK: Oxidative stress and antioxidants in hypertension-a current review. Curr Hypertens Rev 11: 132-142, 2015.

20. Guzik TJ and Touyz RM: Oxidative stress, inflammation, and vascular aging in hypertension. Hypertension 70: 660-667, 2017.

21. Wang D, Strandgaard S, Iversen J and Wilcox CS: Asymmetric dimethylarginine, oxidative stress, and vascular nitric oxide synthase in essential hypertension. Am J Physiol Regul Integr Comp Physiol 296: R195-R200, 2009.

22. Irani K: Oxidant signaling in vascular cell growth, death, and survival: A review of the roles of reactive oxygen species in smooth muscle and endothelial cell mitogenic and apoptotic signaling. Circ Res 87: 179-183, 2000.

23. Argacha JF, Egrise D, Pochet S, Fontaine D, Lefort A, Libert F Goldman S, van de Borne P, Berkenboom G and Moreno-Reyes R: Vitamin D deficiency-induced hypertension is associated with vascular oxidative stress and altered heart gene expression. J Cardiovasc Pharmacol 58: 65-71, 2011.

24. He L, He T, Farrar S, Ji L, Liu T and Ma X: Antioxidants maintain cellular redox homeostasis by elimination of reactive oxygen species. Cell Physiol Biochem 44: 532-553, 2017.

25. Li S, Shao M, Li Y, Li X, Wan Y, Sun X and Cao W: Relationship between oxidative stress biomarkers and visual field progression in patients with primary angle closure glaucoma. Oxid Med Cell Longev 2020: 2701539, 2020.

26. Kong J and Li YC: Effect of ANG II type I receptor antagonist and ACE inhibitor on vitamin D receptor-null mice. Am J Physiol Regul Integr Comp Physiol 285: R255-R261, 2003.

27. Tomaschitz A, Pilz S, Ritz E, Grammer T, Drechsler C, Boehm BO and März W: Independent association between 1,25-dihydroxyvitamin D, 25-hydroxyvitamin D and the renin-angiotensin system: The ludwigshafen risk and cardiovascular health (LURIC) study. Clin Chim Acta 411: 1354-1360, 2010.

28. Xiang W, Kong J, Chen S, Cao LP, Qiao G, Zheng W, Liu W, Li X, Gardner DG and Li YC: Cardiac hypertrophy in vitamin D receptor knockout mice: Role of the systemic and cardiac renin-angiotensin systems. Am J Physiol Endocrinol Metab 288: E125-E132, 2005.

29. Kong J, Qiao G, Zhang Z, Liu SQ and Li YC: Targeted vitamin D receptor expression in juxtaglomerular cells suppresses renin expression independent of parathyroid hormone and calcium. Kidney Int 74: 1577-1581, 2008.

30. Galluzzi L and Green DR: Autophagy-independent functions of the autophagy machinery. Cell 177: 1682-1699, 2019. 
31. Tai S, Hu XQ, Peng DQ, Zhou SH and Zheng XL: The roles of autophagy in vascular smooth muscle cells. Int J Cardiol 211: 1-6, 2016.

32. Salabei JK and Hill BG: Implications of autophagy for vascular smooth muscle cell function and plasticity. Free Radic Biol Med 65: 693-703, 2013.

33. Justin Rucker A and Crowley SD: The role of macrophages in hypertension and its complications. Pflugers Arch 469: 419-430, 2017.

34. Dong Q, Xing W, Fu F, Liu Z, Wang J, Liang X, Zhou X, Yang Q Zhang W, Gao F, et al: Tetrahydroxystilbene glucoside inhibits excessive autophagy and improves microvascular endothelial dysfunction in prehypertensive spontaneously hypertensive rats. Am J Chin Med 44: 1393-1412, 2016.

35. Dong Q, Xing W, Su F, Liang X, Tian F, Gao F, Wang S and Zhang H: Tetrahydroxystilbene glycoside improves microvascular endothelial dysfunction and ameliorates obesity-associated hypertension in obese ZDF rats via inhibition of endothelial autophagy. Cell Physiol Biochem 43: 293-307, 2017.
36. Mondaca-Ruff D, Riquelme JA, Quiroga C, Norambuena-Soto I, Sanhueza-Olivares F, Villar-Fincheira P, Hernández-Díaz T, Cancino-Arenas N, San Martin A, García L, et al: Angiotensin II-regulated autophagy is required for vascular smooth muscle cell hypertrophy. Front Pharmacol 9: 1553, 2018.

37. Wang ZV, Rothermel BA and Hill JA: Autophagy in hypertensive heart disease. J Biol Chem 285: 8509-8514, 2010.

38. Zhou L, Ma B and Han X: The role of autophagy in angiotensin II-induced pathological cardiac hypertrophy. J Mol Endocrinol 57: R143-R152, 2016. International (CC BY-NC-ND 4.0) License. 